\title{
The Bransford-Franks linear effect: Integration or artifact?*
}

\author{
STUART KATZ, BEVERLY ATKESON, and JOANNE LEE \\ University of Georgia, Athens, Georgia 30602
}

\begin{abstract}
It was hypothesized that the Bransford-Franks linear effect is an artifact of the method of presentation of stimulus sentences and is unrelated to semantic processes. Ss were given sentences containing the same information in one of two ways. In a control condition, which was identical to the procedure used in earlier research, overlapping combinations of ideas were presented during learning and recognition; in an experimental condition, ideas were presented one at a time. Results demonstrated that one-idea sentences received significantly higher recognition confidence ratings in the experimental condition, thus supporting the artifact interpretation. It was proposed that Ss assign recognition confidence ratings based on the probability that a sentence containing a certain number of ideas could have occurred in acquisition.
\end{abstract}

Recently Bransford and Franks $(1971,1972)$ carried out a series of experiments on the integration of semantic information in sentences. Their findings revealed (a) that individuals are unable to distinguish between closely related sentences which they heard during an acquisition period and those not heard and (b) that reported recognition of these sentences is a positive linear function of their "sentence complexity," i.e., the number of simple ideas contained in them. (This second finding will hereafter be referred to as the "linear effect.") On the basis of these findings, the authors argued that people spontaneously construct and store in memory a unified semantic representation based on a series of related linguistic inputs; little or no information about specific sentences or specific ideas isolated from the complete representation is retained. To account for the linear effect, they also maintained that recognition of new inputs depends on the number of ideas which the new inputs exhaust in the wholistic representation. The larger the number of ideas in the new input, the smaller its deviation from what has been stored in memory and, hence, the more familiar it is.

Other studies, however, have not entirely supported these arguments. For example, Reitman and Bower (1973) used the Bransford-Franks procedure with strings of letters or digits instead of sentences. They found, among other things, (a) that Ss could indeed distinguish between strings presented in acquisition ("old" strings) and those not presented ("new" strings), (b) that a significant linear relationship occurred between number of items constituting a string and recognition ratings, even though natural language materials were not used, and (c) that this linear effect occurred separately for both old and new strings. In other relevant studies, Katz (1971) and Katz and Gruenewald (1973) found a significant linear effect using either highly abstract

*This research was supported by a grant to the first author from the Small Grants Research Program at the University of Georgia (10-03-RR005-004). sentences (e.g., The original event created an unusual state in the structure of the system) or "meaningless" sentences (e.g., The soto which tehoed inow the feexteva voneed the tioc len ic the froo ab the adex). And finally, Katz (1973) found that the linear effect disappeared entirely when Ss were asked to recognize whether sentences meant exactly the same thing as sentences seen in acquisition (and not whether they had actually experienced such sentences, as in the original research). The result of this last study was unexpected, since the "meaning" instructions were considered more appropriate to a test of the Bransford-Franks model than the original instructions.

For purposes of the present study, these findings demonstrate two things. First, they show that the linear effect is not solely a function of semantic processes, because, on the one hand, the effect occurs when the stimulus materials contain little or no semantic information, while, on the other hand, the effect can be made to vanish even when such information is available under the most appropriate conditions. Second, the results weaken the hypothesis that the linear effect reflects the construction and storage of integrated structures, whether semantic or not. As the Reitman and Bower data show, the effect occurs even under conditions in which Ss can discriminate the new from the old. Thus, virtually all old strings which formed only a part of an integrated structure received much higher ratings than the wholistic structure itself, if that structure happened to be new.

It would appear, then, that the linear effect is unrelated to the explanation that Bransford and Franks offered. The present study attempts to identify that aspect of the Bransford-Franks paradigm which may be responsible for the effect. It is proposed here that the finding is due to the method of presentation of stimulus materials. Let us examine, for the moment, the standard procedure used in these experiments. Typically, complex sentences such as The rock which rolled down the 
mountain crushed the tiny hut at the edge of the sea are analyzed into four simple declarative sentences, each of which expresses a single semantic idea. The simple one-idea sentences are then recombined into other sentences containing two, three, or all four of the ideas. A subset of the sentences thus generated is then used in acquisition (see Procedure for further details). This method assures the redundant, overlapping presentation of all the ideas in any complex sentence and, hence, the integration of its semantic content. However, the method also appears to add a new dimension to the task of recognizing such sentences. Ss may also learn during acquisition that the ideas contained in the sentences appear in various combinations and that the number of combinations varies across levels of sentence complexity. Therefore, they may be assigning ratings based, in part, on some sort of guessing strategy involving the combinatorial properties of the acquisition sentences. For present purposes, the details of such a strategy need not be elaborated, although one possible strategy is described in the discussion section. It is sufficient to point out here that, from a procedural perspective, the linear effect may ultimately be a result of the specific way ideas were communicated in acquisition, and nothing more.

Considerations of this sort suggested a very simple experiment, the outcome of which would support either the Bransford-Franks model or the procedural artifact interpretation proposed here. Ss were given sentences under two procedures which differed in the way the same information was presented. In a control condition, Ss were given the standard procedure in which one-, two-, three-, and four-idea sentences were presented. In the experimental condition, Ss were given only one-idea sentences. The integration of the ideas into complete semantic representations is possible in both conditions (though, perhaps, more difficult in the experimental condition) since even one-idea sentences contain semantic links to other sentences. If the Bransford-Franks hypothesis is correct, $S$ s in both conditions should construct wholistic representations, store them, and later utilize them as a basis for recognizing new sentences. Specifically, one-idea sentences should receive the lowest recognition ratings since, according to the model, they "exhaust" the smallest amount of information in the unified representation. Furthermore, the results should not differ between the experimental and control conditions. If, however, the linear effect is due to the particular method of presentation of acquisition sentences, then the recognition of one-idea sentences should be different in the two conditions. In the control condition, which is simply a replication of the original research, one-idea sentences should receive the lowest ratings relative to other sentences, as has been found in the earlier studies. In the experimental condition, however, $S$ s are, according to this hypothesis, no longer required to decide whether a particular idea appeared alone or in combination with other ideas. If we assume that Ss are, nonetheless, completely familiar with the ideas, the ratings for one-idea sentences should be significantly higher than their counterparts in the control condition.

Before either of the above conclusions can be drawn, it is necessary to demonstrate that $S$ s in both conditions understand the relationships among ideas in each of the unified representations; i.e., Ss must be able to know what ideas go with what. Without such understanding, of course, the spontaneous integration of the ideas cannot take place. In order to evaluate Ss' knowledge of these relationships, two other groups were given the experimental and control acquisition procedures, respectively, and then directly tested for such knowledge. Both groups were given a special class of sentences called "noncases," employed first by Bransford and Franks (1971). The semantic representations of noncases are incompatible with the meaning of the unified representations and should be rejected if Ss understand the interrelationships among ideas. The two groups must be comparable in detecting noncases before any firm conclusion regarding the competing hypotheses can be made.

\section{METHOD}

\section{Subjects}

Ss were 48 volunteers from two undergraduate psychology courses at the University of Georgia.

\section{Materials}

Stimulus materials consisted of a set of four complex embedded English sentences, each containing four unambiguous ideas. Three of the four complex sentences were similar to those used by Bransford and Franks (1971). They are as follows: (a) The rock which rolled down the mountain crushed the tiny hut at the edge of the sea, (b) The barking dog chased the brown cat which jumped on the girl, (c) The ants in the kitchen ate the sweet jelly which was on the newspaper, (d) The thunder crashing through the valley shook the tinkling bells in the corner. Each complex sentence was analyzed into four simple declarative sentences. These latter sentences expressed a single semantic idea in appropriate grammatical form. Thus, The rock which rolled down the mountain crushed the tiny hut at the edge of the sea was partitioned into The rock rolled down the mountain, The rock crushed the hut. The hut was tiny, and The hut was at the edge of the sea. The simple declarative sentences, or ONEs, were then recombined into new sentences expressing two ideas (TWOs), three ideas (THREEs), or four ideas (FOURs). The sentences generated by the partitioning and recombining procedure will be referred to as an "idea group." In addition, four "noncases" were constructed. A noncase was defined as a FOUR in which relationships among grammatical units were changed so as to change the meaning of the sentence. The noncases were (a) The barking dog which chased the brown cat jumped on the girl, (b) The ants on the newspaper ate the sweet jelly which was in the kitchen, (c) The rock which rolled down the mountain shook the tinkling bells in the corner, (d) The barking dog chased the brown cat which was on the newspaper.

(1) Control Condition. The acquisition list for this condition contained a single ONE. TWO. THREE, and FOUR from each idea group, resulting in 16 sentences. Whenever there were more than one to choose from, selection was carried out randomly. With the constraint that no two sentences from the same idea group should appear consecutively, the 16 sentences were 
randomized for presentation during acquisition.

The recognition list contained the same sentences as the acquisition list, although the sentences appeared in a different, randomly generated order.

(2) Experimental Condition. In this condition both the acquisition and recognition lists were composed exclusively of the ONEs in each idea group. Thus, 16 sentences appeared on each list, as in the control condition. Again, no two sentences from the same idea group appeared consecutively and a different random order of ONEs was used for acquisition and recognition, respectively.

\section{Procedure}

With the exception of the type of sentence lists constructed, the procedure closely paralleled that used in Bransford and Franks (1971) for both experimental and control conditions. The experimental session was carried out in two phases, acquisition and recognition.

(1) Acquisition. Acquisition involved an incidental learning task. In addition to a standard set of instructions, Ss were also told that sentences they were about to hear were related. This was done to aid integration. Ss, in groups of two or three, first listened to sentences read by the $E$; each sentence was then followed by a 4- to 5-sec interval in which Ss counted aloud the number of fingers on the E's right hand. After the interpolated task, Ss wrote down what they heard. This procedure was repeated for each of the 16 sentences on the acquisition list. Appropriate counterbalancing was used to control for possible order effects in list presentations. When the list was completed, Ss rested for $5 \mathrm{~min}$ without conversing. During acquisition no mention was made of any recognition test.

(2) Recognition. During recognition Ss were told that a new set of sentences would be read to them and that these sentences were closely related to the set of sentences presented in acquisition. Their task was to indicate which sentences they had actually heard before and which ones they had not. For each sentence Ss indicated whether or not they recognized it by first marking a YES-NO scale and then a five-point confidence scale ranging from "very low" to "very high" confidence. This procedure was repeated for each of the recognition sentences.

(3) Evaluating Integration. As in earlier studies, noncases were used to evaluate Ss' ability to distinguish among sentences with ideas that were either consistent or not consistent with the wholistic representation established in acquisition. However, unlike earlier studies, noncases could not be inserted into the experimental condition recognition list because Ss experienced only ONEs during acquisition. As Bransford and Franks (1972) demonstrated, Ss also retain memory for the "style" of sentences they learned, so that noncases would certainly be rejected on that basis alone; they were much different in structure from the ONEs. Therefore, two additional groups of Ss were used to separately evaluate their ability to detect deviations in meaning from the complete semantic representations. These groups were given the experimental and control acquisition lists, respectively. They were then presented with eight sentences, four noncases and four FOURs. The Ss were asked simply to rate the sentences according to whether they were "consistent" with sentences they heard earlier. In this way, Ss were expected to attend to meaning only, ignoring stylistic differences. Instructions similar to these were effectively used elsewhere (Katz, 1973). As in the recognition conditions for the main part of the experiment, Ss rated sentences by marking YES-NO and confidence scales.

\section{RESULTS}

\section{Data on Sentence Recognition}

The dependent measure used in the present experiment was mean recognition confidence score, determined as follows: Each S's response to the YES-NO and confidence scales for each sentence were converted into a single rating. A plus ( + ) was assigned to a YES response and a minus $(-)$ to a NO response. The + or was multiplied by the S's numerical response to the confidence scale for that sentence. This resulted in an 11-point recognition confidence scale ranging from -5 to +5 . In the control condition, each of these ratings was pooled for FOURs, THREEs, TWOs, and ONEs. In the experimental condition, all ratings were pooled into a single group, since, of course, all the sentences Ss rated were old ONEs.

(1) Replication of Linear Effect. Mean recognition confidence ratings in the control condition were 3.48 , $2.96,2.04,-1.10$ for FOURs, THREEs, TWOs, and ONEs, respectively. The data for the control condition were cast into a single-factor repeated-measure design with "sentence complexity" (FOURs, THREEs, TWOs, and ONEs) as the factor. An overall analysis of variance yielded a significant $F$ ratio for sentence complexity. A trend analysis was carried out on the same data and a significant linear trend was found for sentence complexity $[F(1,33)=15.42, p<.001]$. Therefore, the control condition replicates the findings of Bransford and Franks (1971).

(2) Comparison of Experimental and Control Conditions. The crucial comparison in the present study was between the ratings for all sentences in the experimental condition (these were entirely ONEs) and the ratings for ONEs in the control condition. The means for ONEs in the experimental and control conditions were 3.31 and -1.10 , respectively. The difference between these means was highly significant $[\mathrm{t}(22)=4.70, \mathrm{p}<.001]$. Thus, sentences in the experimental condition received ratings significantly higher than sentences in the control condition.

\section{Evaluating Integration: Noncases}

The means for noncases in the experimental and control conditions were -1.59 and -1.86 , respectively, and they were not significantly different $[t(22)=0.52$, $p<.50]$. Therefore, the null hypothesis that recognition confidence ratings for experimental Ss do not differ from those for control Ss cannot be rejected.

\section{DISCUSSION}

It was hypothesized that the linear effect, i.e., the linear relationship between recognition confidence ratings and the number of ideas in recognition sentences, is a function of the mode of presentation of acquisition sentences, not of semantic processes. To test this hypothesis, two different types of acquisition lists were used within the standard Bransford-Franks format. In a control condition, sentences were presented that varied in the number of ideas each contained. In the experimental condition, only ONEs, or single-idea, 
sentences were presented. According to Bransford and Frank $(1971,1972)$, either method should, in principle, result in the formation of wholistic semantic representations which, in turn, should provide the basis for recognition of new sentences. Thus, recognition confidence ratings for old ONEs ought to be identical regardless of which acquisition list is employed. According to the procedural artifact hypothesis proposed here, ONEs in the experimental condition should be significantly higher because Ss are no longer required to remember what the precise combinations of ideas were in acquisition. They need only remember whether they saw the ideas at all. The results unequivocally support the artifact hypothesis; ONEs in the experimental condition received high positive recognition confidence ratings comparable to the highest ratings in the control condition and significantly higher than old ONEs in the control condition. Furthermore, since Ss who experienced either the experimental or control acquisition lists did not differ significantly in their ability to detect noncases, the difference in recognition of ONEs cannot be attributed to differences in degree of integration between the two conditions.

Perhaps, however, the results are due to other differences between the experimental and control conditions. Three are apparent. First, acquisition and recognition sentences differ in length for the two conditions. In the experimental condition, sentences are short and roughly of comparable length; in the control, sentences vary in length, some being quite long (the FOURs) and others short (the ONEs). Second, the two conditions differ in the frequency with which simple ideas were presented in acquisition. In the experimental condition, each simple idea appeared only once, while, in the control, each appeared at least twice and often three times. Neither of these differences, length nor frequency, can be plausibly connected to the results. In fact, the argument could be made that they would lead to findings opposite those observed here. However, a third difference could reasonably account for the findings. It will be recalled that the mean recognition confidence rating for ONEs was based on 16 sentences in the experimental condition and on only four in the control. Singer and Rosenberg (1973) have recently shown that ratings among ONEs differ considerably as a function of the grammatical relations existing between simple ideas and the FOURs from which they are derived. Thus, for example, ONEs which express the subject-object relation of the main clause of a FOUR ("MAINs") are likely to receive much higher ratings than ONEs which do not contain this relation. If, in the present study, ONEs in the control condition contain relatively unimportant relations, their ratings might be lower than ONEs in the experimental condition (four of which contain the important MAIN relation) for this reason. Inspection of the ONEs in the control condition revealed, however, that three contained the important MAIN relation. Therefore, the difference in ratings between ONEs in the two conditions cannot be accounted for in this way. ${ }^{1}$ Since it could still be argued that other unspecified differences between experimental and control ONEs are responsible for the findings, the ratings for the control ONEs were compared to the ratings for the same four ONEs in the experimental condition. The means were 3.44 for the experimental condition and -1.10 for the control, and their difference was highly significant $[\mathrm{t}(22)=4.58$, $\mathrm{p}<.001]$. Therefore, even when identical ONEs in the two conditions are compared, the results are the same. In sum, there appears to be no way to account for the results other than by the differences in the way ideas were combined in the two conditions.

Though the present study suggests the probable soirce of the linear effect, no precise explanation has been offered as to why the particular acquisition procedure used by Bransford and Franks should invariably produce such a relationship. A relatively simple hypothesis offered here is that Ss assign ratings based on the probability that a sentence having a particular number of ideas could have occurred in acquisition. They would do this, presumably, by estimating the total possible number (or "set size") of sentences of varying sentence complexity and then formulating a probability based on the inverse of that set size. The linear effect itself can, thus, be easily explained: Since the maximum set size is greatest for ONEs and least for FOURs, the probability estimates and, hence, recognition confidence ratings would yield a reverse ordering, i.e., ratings would be greatest for FOURs and least for ONEs. The hypothesis can also explain other related findings, including those where the positive linear relationship is not obtained. Two investigations are mentioned here. In the Reitman and Bower (1973) study described earlier, a "random" condition was included in which strings of elements (letters or digits) of differing lengths were generated by randomly grouping elements without regard to order or type of element. Under these circumstances, all permutations and combinations of elements are permissible, so that the set size for FOURs becomes the largest and the set size for ONEs the smallest. If our hypothesis is correct, ratings for those strings should yield a "reverse" linear effect; those for ONEs should be highest and FOURs smallest. This is exactly what Reitman and Bower (1973) found. Bransford and Franks (1972) report a related study in which the basic ideas of several wholistic representations were presented "unconstrained" in acquisition, i.e., the basic ideas were combined in ways that cut across wholistic representations. This manipulation increases the potential number of sentences of higher complexity relative to those of lower and, according to our hypothesis, should change the slope of the sentence complexity curve so that ratings of sentences of greater complexity decrease, while those of lesser complexity increase. Results of the study showed the slope of the 
curve to be virtually zero, a change in a direction consistent with the hypothesis. We should point out that the curve would not be expected to reflect a completely reverse ordering in the ratings, as in the Reitman and Bower (1973) study, because the unconstrained condition permitted only a few additional combinations (and no permutations) of basic ideas relative to the standard procedure. As a result, the set sizes of higher complexity sentences were much smaller than those of the higher complexity letter or number strings in Reitman and Bower (1973).

It remains for us to consider the general question of why Ss might assign ratings using this "statistical" method rather than attempt a precise identification of each sentence during recognition. In fact, Ss probably do try to make exact identifications. However, except in the cases where there are major changes in meaning (as with noncases) or in syntactic style (Katz, 1973), or perhaps where the amount of information contained in the stimulus material is relatively small (Reitman \& Bower, 1973), precise recognition becomes especially difficult. Therefore, Ss must resort to some other way of assigning ratings. The strategy we have described seems most plausible because, while the presentation of redundant overlapping ideas is difficult for Ss vis-a-vis the recognition of particular sentences, that very same procedure is quite clear in suggesting the way in which the various simple ideas go together and, as a consequence, how sentences expressing part or all of a wholistic idea can be generated. Once Ss know this, it is a relatively simple matter for them to formulate the requisite probability estimates and the corresponding recognition confidence ratings.

\section{REFERENCES}

Bransford, J. D., \& Franks, J. J. The abstraction of linguistic ideas. Cognitive Psychology, 1971, 2, 331-350.

Bransford, J. D., \& Franks, J. J. The abstraction of linguistic ideas: A review. Cognition. International Journal of Cognitive Psychology, 1972, 1, 221-249.

Katz, S. The role of representational information and instructional set in the integration of semantic ideas. Unpublished doctoral dissertation, University of Michigan, 1971.

Katz, S. Role of instruction in abstraction of linguistic ideas. Journal of Experimental Psychology, 1973, 98, 79-84.

Katz, S., \& Gruenewald, P. The abstraction of linguistic ideas in "meaningless" sentences. Unpublished manuscript, University of Georgia, 1973 .

Reitman, J. S., \& Bower, G. H. Storage and later recognition of exemplars of concepts. Cognitive Psychology, 1973, 4, 194-206.

Singer, M., \& Rosenberg, $S$. The role of grammatical relations in the abstraction of linguistic ideas. Jourmal of Verbal Learning \& Verbal Behavior, 1973, 12, 273-284.

\section{NOTE}

1. Indeed, it would be expected that control ONEs would show the higher ratings, since 3 out of 4 of them contain the MAIN relation, as compared to 4 out of 16 for the experimentai ONEs.

(Received for publication March 11, 1974; accepted April 6, 1974.) 